\title{
An Efficient Image Fusion of Visible and Infrared Band Images using Integration of Anisotropic Diffusion and Discrete Wavelet Transform
}

\author{
Binal Panchotiya, Dippal Israni, and Ritesh Patel
}

\begin{abstract}
Image fusion is a technique that combines two source images to generate more informative target image. It plays a vital role in medical image investigation, military, navigation, etc. visible images offer efficient texture detail with high spatial resolution. In contrast, based on the radiation difference infrared images are able to differentiate target from their background. There are many algorithms that helps in preserving the edges of image like Bilateral filter, Anisotropic Diffusion (ADF). This paper proposes a novel approach by integrating Anisotropic Diffusion and Karhunen-Loeve (KL) Transformation with Discrete Wavelet Transform (DWT). In proposed Method, DWT decomposes into four sub-bands. ADF is applied on approximation sub-band and absolute maximum selection is applied on other three sub-bands. ADF decomposes the image into detailed layer and base layer. Base layer and Detailed layer are calculated using Kl- Transformation and linear combination respectively. Once fusion is done, inverse DWT is applied on all sub-bands. The experimental outcomes depict that the offered approach result with sharp edges of the image. The proposed algorithm is evaluated on standard dataset Like Duine_Sequence, Tree_Sequence, Street dataset. Standard metrics like Average Gradients and Spatial Frequency metrics are used to evaluate the performance of the image. The results depict that the proposed approach outperforms state of the art approaches on various datasets.
\end{abstract}

Index Terms - Image fusion, Infrared and Visible image, DWT, Anisotropic Diffusion.

\section{INTRODUCTION}

Image fusion is a procedure that targets to integrate two images attained by one or more different kinds of sensor to achieve an informative image. This information is useful in decision making [1]. In recent years, research on image fusion has been growing rapidly. The enhanced vision system, medical diagnosis, robotics, military, surveillance, object identification [2] [3], classification and change detection are the application

Manuscript received April 18, 2019; revised November 6, 2019. Date of publication March 9, 2020. Date of current version March 9, 2020.

B. Panchotiya and R. Patel are with the U and P U. Patel Department of Computer Engineering, Chandubhai S. Patel Institute of Technology, CHARUSAT. D. Israni is with the Information Technology Department, R. C. Technical Institute.

E-mails: binalpanchotiya8520@gmail.com, dippalisrani90@gmail.com, riteshpatel.ce@charusat.ac.in.

Digital Object Identifier (DOI): 10.24138/jcomss.v16i1.760 of image fusion [4].

Image fusion can be applied to various source images like infrared and visible bands, Panchromatic (PAN) and Multispectral (MS) bands. Infrared and visible band images have many aspects.

Visible band images represent the spatial information of the fused image and also contains the texture detail of the scene and edges details [5]. In contrast, infrared images have an advantage over the uncovering of the fused image. It can also work efficiently under the illness of night vision, bad weather and weak light [5]. The focus of this paper is on the fusion of Visible and Infrared band images.

Image fusion can be categorized as depicted in Fig. 1

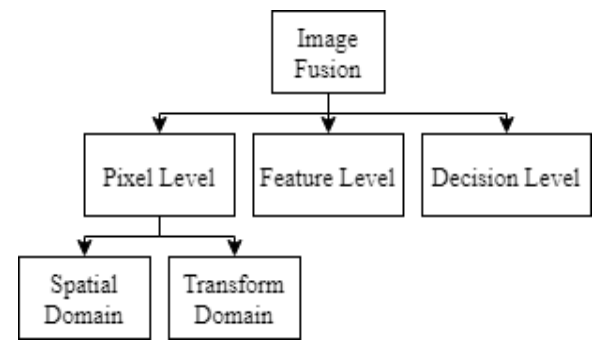

Fig. 1. Classification of Image Fusion

Pixel-level image fusion works directly on the spatial information (pixel intensity) of the image [6]. In Feature-level image fusion, features are extracted from the source image [6]. Decision-level image fusion is a high level of image fusion, integrates the outcomes from multiple technique to proceeds a final fused decision [7]. Pixel-level image fusion is further categorized into transform domain [8] and spatial domain [8]. Image fusion in spatial domain deal with pixel intensity of the input image. In contrast, image fusion in transform domain works with the frequency representation of an image.

Image fusion is an essential area in an information science. Over the past decade, various different algorithms are introduced, each having their own methodology. Edgepreserving smoothing is an image processing procedure that smooth away from noise or texture and retaining the sharp edges. Recently, there are many edge preserving techniques used for image fusion that includes weighted least square filter 
[9], guided image filter [10], 3-D anisotropic diffusion [11], cross bilateral filter [12], bilateral filter [13], etc. All these techniques first segment image into base layer and then into detailed layer. Final fused image can be generated after applying fusion rule on base and detail layers. Anisotropic diffusion gives adaptability of smoothing the image while preserving the high frequency [14]. D. Bavirisetti et al. proposed anisotropic diffusion process [11], [15] that gives a sharp and informative image at homogeneous domain. In Image Fusion, the two state of the art approaches includes Anistotropic Diffusion and through DWT. Both techniques have their limitations. To overcome this challenge, this paper proposed a novel technique of integrating two state of the art approaches.

The rest of this work is organized as follows. An overview on image fusion techniques is described in section II. The proposed method is described in section III. Section IV showcases the performance of the proposed approach on standard datasets evaluated it by comparing it with other competitive methods. Section V contains a conclusion.

\section{OVERVIEW OF IMAGE FUSION METHOD}

An outline of Image Fusion method using state of the art anisotropic diffusion is depicted in Fig. 2.

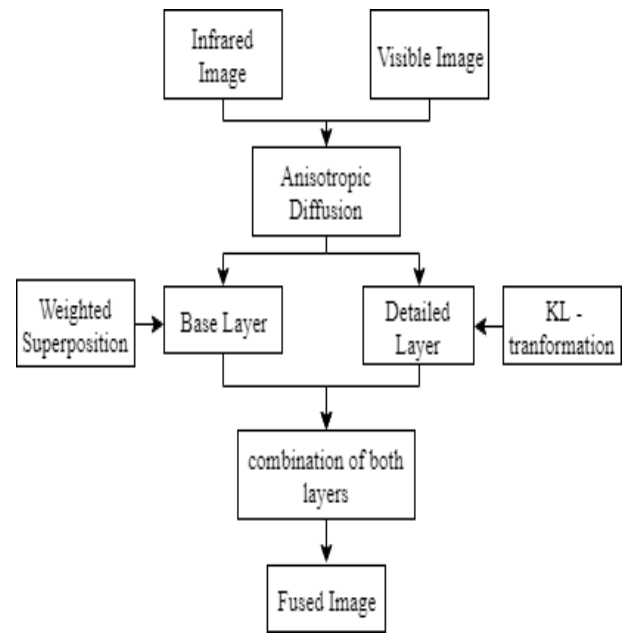

Fig. 2. Schematic of ADF algorithm and KL Transformation apply on image fusion

Fig. 2 represents decomposition of source images into base layer and detailed layer via ADF. Firstly, ADF extract the base layer of the source images. Detailed layer is obtained by subtracting base layer from source images. Base layer and detailed layer calculated with the Kl - Transformation and linear combination respectively. Finally calculate the inverse of image and get the fused image.

The ADF [14] is control the diffusion of image by using flux function. For that the equation of $\mathrm{ADF}$ is given below:

$$
\begin{gathered}
\frac{\partial I}{\partial x}=\operatorname{div}(\mathrm{c}(\mathrm{x}, \mathrm{y}, \mathrm{t}) \nabla I) \\
I_{t}=\nabla c \cdot \nabla I+\mathrm{c}(\mathrm{x}, \mathrm{y}, \mathrm{t}) \nabla I
\end{gathered}
$$

where, $I=$ image, $\nabla=$ gradient operator, $\Delta=$ laplacian operator, $t=$ iteration or scale or time, $\mathrm{c}(\mathrm{x}, \mathrm{y}, \mathrm{t})$ is the diffusion coefficient.

Eq. 2 referred as a heat equation. The solution for this PDE is calculated by using Forward Time Central Space (FTCS).

$$
\begin{gathered}
I_{i, j}^{t+1}=I_{i, j}^{t}+\lambda\left[c_{E} \cdot \bar{\nabla}_{E} I+c_{W} \cdot \bar{\nabla}_{W} I+c_{N} \cdot \bar{\nabla}_{N} I\right. \\
\left.+c_{S} \cdot \bar{\nabla}_{S} I\right]_{i, j}^{t}
\end{gathered}
$$

where, $\lambda$ is constant between $0 \leq \lambda \leq \frac{1}{4}$. $I_{i, j}^{t+1}$ is coarser resolution image at $\mathrm{t}+1$ Image. $I_{i, j}^{t}$ is previous coarser image. $\bar{\nabla}_{E}, \bar{\nabla}_{W}, \bar{\nabla}_{N}, \bar{\nabla}_{S}$ indicates nearest-neighbour differences and is calculated as below in East, West, North and South directions respectively.

$$
\begin{gathered}
\nabla_{E} I_{i, j}=I_{i, j}+1-I_{i, j} \\
\nabla_{W} I_{i, j}=I_{i, j}-1-I_{i, j} \\
\nabla_{N} I_{i, j}=I_{i-1, j}-I_{i, j} \\
\nabla_{S} I_{i, j}=I_{i, j}+1-I_{i, j}
\end{gathered}
$$

Likewise, flux or conduction coefficients $c_{E}, c_{W}, c_{N}, c_{S}$ are updated during every iteration in different directions is defined as below:

$$
\begin{aligned}
& C_{E_{i, j}}^{t}=g\left(\|(\nabla I)_{i, j+\frac{1}{2}}^{t}||\right)=g\left(\left|\bar{\nabla}_{E} I_{i, j}^{t}\right|\right) \\
& C_{W_{i, j}}^{t}=g\left(\|(\nabla I)_{i, j-\frac{1}{2}}^{t}||\right)=g\left(\left|\bar{\nabla}_{W} I_{i, j}^{t}\right|\right) \\
& C_{N_{i, j}}^{t}=g\left(\left\|(\nabla I)_{i-\frac{1}{2}, j}^{t}\right\|\right)=g\left(\left|\bar{\nabla}_{N} I_{i, j}^{t}\right|\right) \\
& C_{S_{i, j}}^{t}=g\left(\left\|(\nabla I)_{i+\frac{1}{2}, j}^{t}\right\|\right)=g\left(\left|\bar{\nabla}_{S} I_{i, j}^{t}\right|\right)
\end{aligned}
$$

Here, $g(\cdot)$ is decreasing function with $g(0)=1$. Distinct functions can be used for $\mathrm{g}(\cdot)$. Perona and Malik [12] recommended two function as below:

$$
\begin{aligned}
& \mathrm{g}(\nabla I)=\frac{1}{1+\left(\frac{\|\nabla I\|}{k}\right)^{2}} \\
& \mathrm{~g}(\nabla I)=e^{-\left(\frac{|\nabla \nabla| \mid}{k}\right)^{2}}
\end{aligned}
$$

In both functions, constant $\mathrm{k}$ is used for determine the validity of a region boundary based on its edge strength.

One of the most popular techniques is wavelet transform based techniques. Wavelet theory is frequency based techniques that enhances the result of image by changing the low-frequency and high frequency parameter. DWT [16], [17], [18], is a most popular technique in information science that is used to retrieve multi-resolution of image.

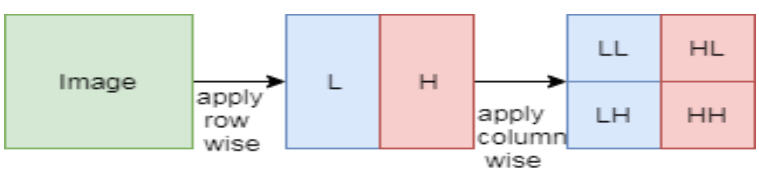

Fig. 3. Decomposition of image 
As shown Fig. 3 DWT decomposes image in four sub bands. Initially, image is decomposed using row-wise DWT which results into low frequency and high frequency details. Both are further decomposed using column-wise DWT that results into four sub bands. These four bands are Approximation band (LL), Vertical band (LH), Horizontal band (HL) and Diagonal band $(\mathrm{HH})$.

Fig. 4. illustrates decomposition of images into four sub bands using DWT. An average of LL subband of visible and infrared is computed. Also, maximum of LH, HL and $\mathrm{HH}$ bands are computed of visible and infrared Images. Finally, apply inverse dwt on the image and retrieve final fused image.

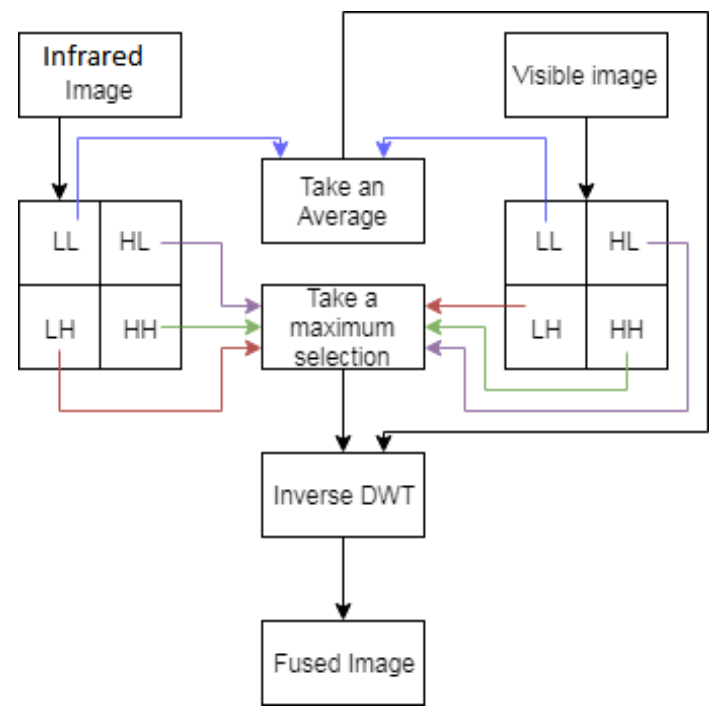

Fig. 4. Image fusion based on DWT

The scaling function $\phi(\mathrm{t})$ and wavelet function $\psi(\mathrm{t})$ of DWT is illustrated as below:

$$
\begin{aligned}
& \emptyset(t)=\sum_{k=-\infty}^{\infty}\left(p_{k}\right) Q(2 t-k) \\
& \varphi(t)=\sum_{k=-\infty}^{\infty}\left(q_{k}\right) Q(2 t-k)
\end{aligned}
$$

The haar wavelet transform is one of the simplest transforms and sequence of rescaled "square-shaped" functions in wavelet family. The scaling function $\phi(\mathrm{t})$ and mother wavelet function $\psi(\mathrm{t})$ of haar wavelet [16], [17], $\mathrm{p}_{\mathrm{k}}$ and $\mathrm{q}_{\mathrm{k}}$ are represented as low pass and high pass filter respectively.

$$
\begin{gathered}
\phi(t)=\left\{\begin{aligned}
-1, & 0 \leq t<1 \\
0, & \text { otherwise }
\end{aligned}\right. \\
\varphi(t)=\left\{\begin{aligned}
1, & 0 \leq t<1 / 2 \\
-1, & 1 / 2 \leq t<1 \\
0, & \text { otherwise }
\end{aligned}\right.
\end{gathered}
$$

\section{PROPOSED APPROACH}

Related work represents functioning of ADF and DWT and recent approaches towards it. By using Partial Differential Equation (PDE), ADF [12] preserves nonhomogeneous regions (edges) and it smooths homogeneous regions. DWT provides multiresolution of image and it also provides perfect image reconstruction. However, ADF individually is not able to preserve the edges while completely removing the noise using the ADF after some extent. Similarly, DWT also has some challenges such as less directional selectivity, shift invariance, aliasing, oscillation of wavelet coefficients This paper proposes an efficient method that overcomes limitations in both state of the art techniques.

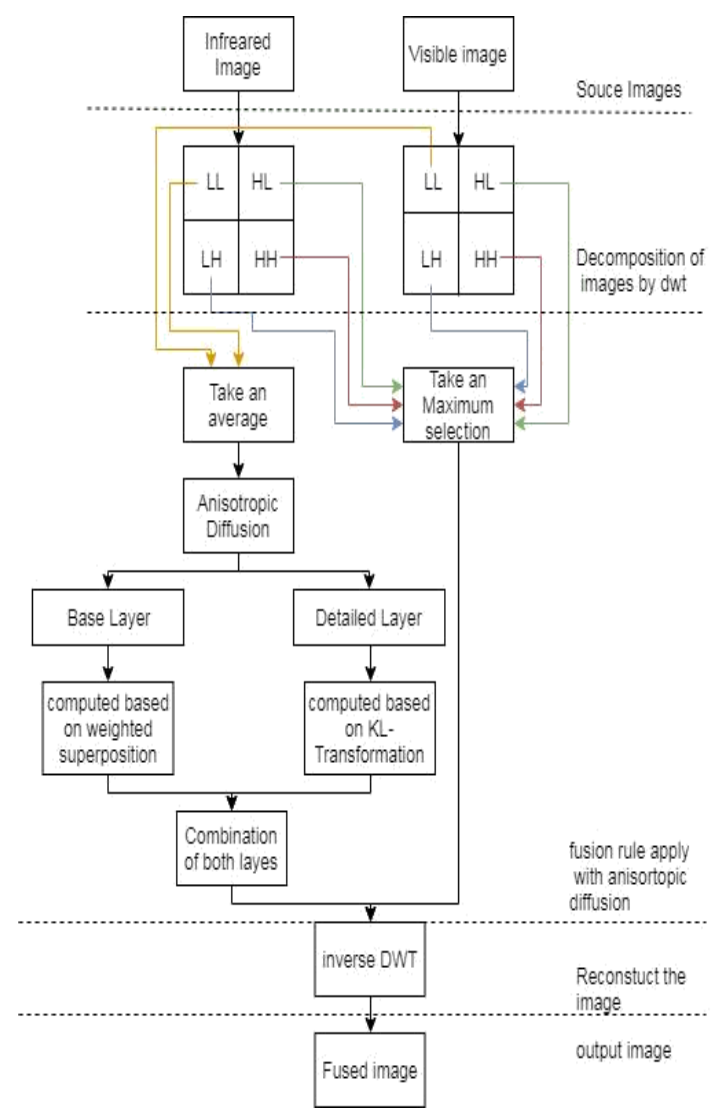

Fig. 5. Image fusion based on DWT

Fig. 5. represents decomposition of two source images using DWT. The image is decomposed into four sub bands. Average is computed of approximation band of two images. Anisotropic diffusion algorithm is applied with KL-transformation on the resulting approximate image. The approximation band and other three sub bands of two images are integrated. Finally, inverse DWT is computed on integrated images to achieve final efficient fused image.

\section{A. Methodology}

In this paper, infrared and visible band images are considered as an input. The size of both input images are computed. Images are resized if the size of the image differs. DWT algorithm is applied on both images. The images are separated into four sub bands HH, LL, LH and HL. LL band describes an approximation details of images. LL sub bands are integrated using average operation on it. Anisotropic diffusion with KL transformation is applied on the resulting image. ADF decomposes Approximation band (LL) of images into the detailed layer and base layer using procedure as shown below. 
The input Approximation band (LL) of images $\left.I_{n}(p, q)\right\}_{n=1}^{N}$ with $\mathrm{R} \times \mathrm{S}$ size all are registered. Now, Anisotropic diffusion is applied on that to get base layers.

$$
b_{n}(\mathrm{p}, \mathrm{q})=\operatorname{anisotropic}\left(I_{n}(\mathrm{p}, \mathrm{q})\right)
$$

where, $b_{n}(\mathrm{p}, \mathrm{q})$ is the $n^{\text {th }}$ base layer and anisotropic $\left(I_{n}(\mathrm{p}, \mathrm{q})\right)$ denotes the anisotropic diffusion process on the $n^{\text {th }}$ input images and $\mathrm{p}$ and $\mathrm{q}$ denotes the pixel values.

To get detailed layer from Approximation band (LL) of images, subtract base layer from input images.

$$
d_{n}(\mathrm{p}, \mathrm{q})=I_{n}(\mathrm{p}, \mathrm{q})-b_{n}(\mathrm{p}, \mathrm{q})
$$

where, $d_{n}(\mathrm{p}, \mathrm{q})$ is the $n^{\text {th }}$ detailed layer of the $n^{\text {th }}$ input image.

Weighted superposition is computed on Base layer. This is retrieved by allocating some weight $w_{n}$ to images. This can be represented as:

$$
B_{n}=w_{n} \sum_{n=1}^{N} b_{n}(\mathrm{p}, \mathrm{q})
$$

where $0 \leq w_{n} \leq 1$ and $\sum w_{n}=1$.

Here, for $\forall \mathrm{n}, w_{1}, w_{2}, w_{3}, \ldots \ldots \ldots w_{N}=\frac{1}{N}$ represents the average of Base Layer.

Calculate detail layers using KL transform [9]. It converts the correlated components into the uncorrelated components [10]. This is computed using following steps.

A.1 ADF algorithm is applied on various Approximation band (LL) of $\mathrm{N}$ images. There are two input images $I_{1}(p, q)$ and $I_{2}(p, q)$ having two detailed layers $d_{1}(p, q)$ and $d_{2}(p, q)$ respectively. These detail layers are placed as column vectors of a matrix $\mathrm{A}$.

A.2 Calculate the covariance matrix $C_{a a}$ of $\mathrm{A}$ in terms of each column as a variable and each row as an observation.

A.3 Calculate Eigen values $C_{1}, C_{2}$ and Eigen vector $V_{1}, V_{2}$ of $C_{a a}$.

$$
V_{1}=\left[\begin{array}{l}
V_{1}(1) \\
V_{1}(2)
\end{array}\right], V_{2}=\left[\begin{array}{l}
V_{2}(1) \\
V_{2}(2)
\end{array}\right]
$$

A.4 Eigen vector $V_{\max }(\mathrm{i})$ is computed corresponding to the large Eigen values $C_{\text {maximum }}=$ maximum $\left(C_{1}, C_{2}\right)$.

A.5 Uncorrelated components $\mathrm{K} L_{1}$ and $\mathrm{K} L_{2}$ are computed as below:

$$
\mathrm{K} L_{1}=\frac{V_{\max }(1)}{\sum_{i} V_{\max }(i)}, \quad \mathrm{K} L_{2}=\frac{V_{\max }(2)}{\sum_{i} V_{\max }(i)}
$$

A.6 The final fuse detailed layer $\mathrm{D}$ is retrieved as below:

$$
\mathrm{D}(p, q)=\mathrm{K} L_{1} d_{1}(p, q)+\mathrm{K} L_{2} d_{2}(p, q)
$$

A.7 For $\mathrm{n}$ detail layer the generalized equation is

$$
D(p, q)=\sum_{n=1}^{N} d_{n}(p, q) K L_{n}
$$

By using a simple linear combination of Base layer and the Detailed layer, final fuse Approximation band (LL) of image is retrieved.

$$
\mathrm{F}=\mathrm{B}+\mathrm{D}
$$

Once the approximate band is integrated with ADF. HL, LH and $\mathrm{HH}$ bands of images are computed. The coefficients of HL, $\mathrm{LH}, \mathrm{HH}$ bands of two images are computed using choose-max rule as below.

$$
\begin{aligned}
\operatorname{Hf}(i, j) & =\left\{\begin{array}{cc}
H L_{1}(i, j) & H L_{1}(i, j) \geq H L_{2}(i, j) \\
H L_{2}(i, j) & \text { Others }
\end{array}\right. \\
\operatorname{Vf}(i, j) & =\left\{\begin{array}{lc}
V L_{1}(i, j) & V L_{1}(i, j) \geq V L_{2}(i, j) \\
V L_{2}(i, j) & \text { Others }
\end{array}\right. \\
\operatorname{Df}(i, j) & =\left\{\begin{array}{lc}
\mathrm{D} L_{1}(i, j) & \mathrm{D} L_{1}(\mathrm{i}, \mathrm{j}) \geq \mathrm{D} L_{2}(\mathrm{i}, \mathrm{j}) \\
\mathrm{D} L_{2}(\mathrm{i}, \mathrm{j}) & \text { Others }
\end{array}\right.
\end{aligned}
$$

Lastly, inverse DWT is applied on fused coefficient to retrieve final fused image.

\section{Simulation Results}

In this paper, the proposed algorithm is evaluated on standard dataset such as tree_sequence [20], duine_sequence [20], and street [21].

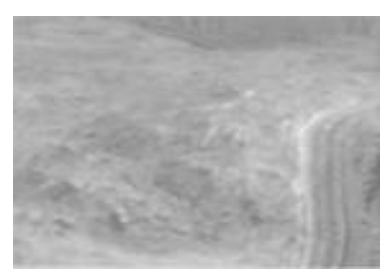

(a)

(c)

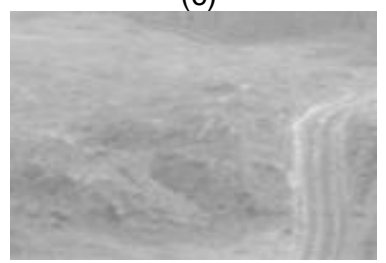

(e)

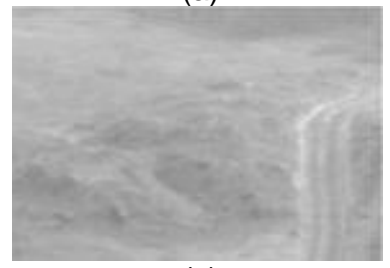

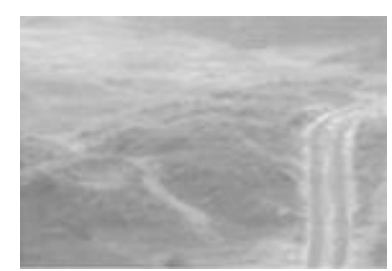

(b)

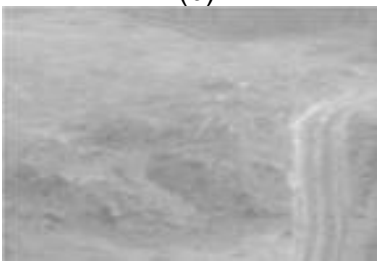

(d)
Fig. 6. Duine_Seuence Dataset: (a) Orignal Infrared Image (b) Original Visible Image (c) Result of DWT (d) Results of Anisotropic. (e) Results of DWT with Anistropic.

The experiments are first listed, compared and simulated in MATLAB $15 \mathrm{~b}$. The datasets contain infrared and visible bands images. Fig. 6., Fig. 7. and Fig. 8. showcases the results by different methods. The results of fused images obtained by new suggested algorithm is compared with existing two fused algorithms on different dataset. These two existing algorithms are discrete wavelet transform algorithm [16]-[18] and Anisotropic algorithm [14]. To validate the proposed method 
standard parameter are used to measure the image quality. These standard parameters are spatial frequency [1] and average gradients [19].

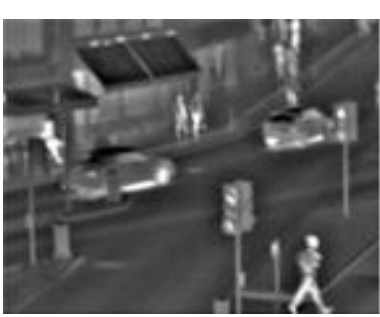

(a)

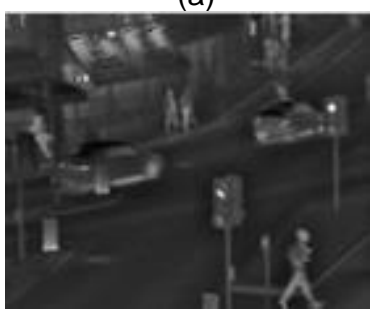

(c)

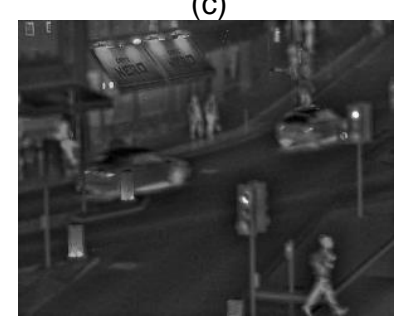

(e)

Fig. 7. Street Dataset: (a) Orignal Infrared Image (b) Original Visible Image (c) Result of DWT.(d) Results of Anisotropic. (e) Results of DWT with Anistropic.

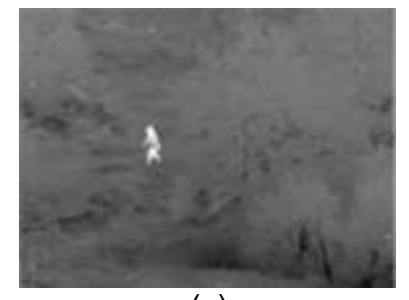

(a)

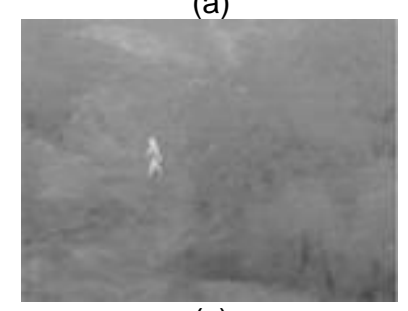

(c)

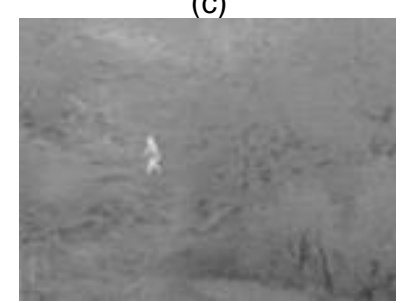

(e)

Fig. 8. Tree_Seuence Dataset: (a) Orignal Infrared Image (b) Original Visible Image (c) Result of DWT.(d) Results of Anisotropic. (e) Results of DWT with

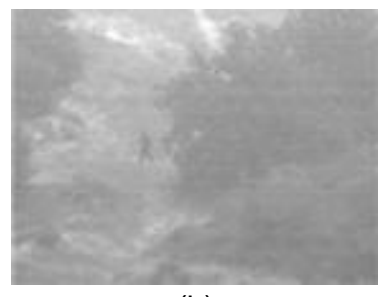

(b)

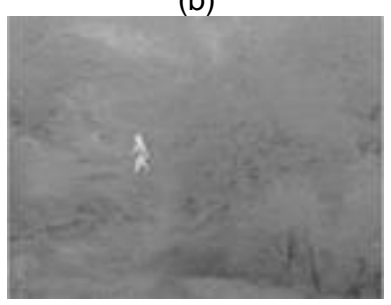

(d) Anistropic.

\section{A. Spatial Frequency}

The spatial frequency (SF) [1] computed as follows

$$
\mathrm{SF}=\sqrt{R F^{2}+C F^{2}}
$$

where, $\mathrm{CF}$ and RF is Column Frequency and Row Frequency respectively.

$$
\begin{gathered}
\mathrm{RF}=\sqrt{\frac{1}{M \times N} \sum_{i=1}^{M} \sum_{J=2}^{N}[F(i, j)-F(i, j-1)]^{2}} \\
\mathrm{CF}=\sqrt{\left.\frac{1}{M \times N} \sum_{i=1}^{M} \sum_{J=2}^{N}[F(i, j)-F(i-1, j)]^{2}\right)}
\end{gathered}
$$

where, $F=$ given image, $(i, j)=$ Pixel Index,$f(i, j)=$ gray value at pixel (i,j), $M=$ number of rows, $N=$ number of column. The huge SF of fused image is more informative in terms of edges and texture.

\section{B. Average Gradients}

The average gradients (AG) [18] computed as follow:

$$
\mathrm{AG}=\frac{1}{M \times N} \sum_{i=1}^{M} \sum_{j=1}^{N}\left[\Delta p f(i, j)^{2}+\Delta q f(i, j)^{2}\right]^{\frac{1}{2}}
$$

where, $\Delta p f$ and $\Delta q f$ is the difference value of pixel in $\mathrm{p}$ or $\mathrm{q}$ direction. $\mathrm{M}$ denotes the number of rows and $\mathrm{N}$ denotes the number of columns. It is used to define the minor changes of detailed contrast and variation of texture information.

\section{Entropy}

Entropy utilizes the basis of information theory to estimate the amount of information contained in a fused image on the basis of information theory The Entropy (ET) [18] can be computed as follows:

$$
E N=-\sum_{l=0}^{L-1} H_{l} \log _{2} H_{l}
$$

where, $L$ represents the occurrences of gray levels and $\mathrm{H}_{1}$ is the normalized histogram of the corresponding gray level in the fused image. The higher value of EN depicts more information enclosed in the fused image. It also represents the efficient performance of the fusion technique.

\section{Mutual Information}

The Mutual Information (MI) [18] can be computed as follow:

$$
M I=M I_{V i s, f u s}+M I_{\text {inf }, f u s}
$$

$\mathrm{MI}_{\text {vis,fus }}$ and $\mathrm{MI}_{\text {inf,fus }}$ represents the information transferred from visible as well as in frared images to the fused image. The MI amongst two random variables can be estimated by the standard Kullback-Leibler measure as follow:

$$
M I_{X, f u s}=\sum_{x, F} H_{X, F}(X, F) \log \frac{H_{X, f u s}(X, F)}{H_{X}(X) H_{F}(F)}
$$


$\mathrm{H}_{\mathrm{X}}(\mathrm{X})$ and $\mathrm{H}_{\mathrm{F}}(\mathrm{F})$ represents the marginal histograms of input image $\mathrm{X}$ and fused image $\mathrm{F}$, respectively. $\mathrm{H}_{\mathrm{X}, \mathrm{F}}(\mathrm{X}, \mathrm{F})$ represents the joint histogram of input image $X$ and fused image $F$. A high MI metric represents that substantial information is transferred from input images to the fused image. This also depicts an efficient fusion performance.

Table I. represents results of the state-of-the-art methods and proposed approach on standard dataset using standard evaluation parameter. Percentage increase indicates the quality increased in percentage of proposed method as in compare with highest state of the art method.

TABLE I

COMPARISON BETWEEN DIFFERENT ALGORITHMS BASED ON EVALUATION MATRICES

\begin{tabular}{|c|c|c|c|c|c|}
\hline $\begin{array}{c}\text { Image } \\
\text { Dataset }\end{array}$ & PM & DWT & ADF & $\begin{array}{c}\text { ANI } \\
\text { DWT }\end{array}$ & $\begin{array}{c}\% \\
\text { Increase }\end{array}$ \\
\hline \multirow{4}{*}{$\begin{array}{c}\text { Duine_ } \\
\text { Sequence } \\
\text { Dataset }\end{array}$} & SF & 3.0414 & 1.9895 & 3.0592 & 0.585257 \\
\cline { 2 - 6 } & AG & 21.6511 & 10.2932 & 21.8092 & 0.730217 \\
\cline { 2 - 6 } & ET & 5.8044 & 5.7689 & 5.8057 & 0.022397 \\
\hline \multirow{4}{*}{$\begin{array}{c}\text { Tree_ } \\
\text { Sequence } \\
\text { dataset }\end{array}$} & SF & 11.6088 & 11.5378 & 11.6114 & 0.022397 \\
\cline { 2 - 6 } & AG & 19.7909 & 12.1065 & 19.7995 & 0.043454 \\
\cline { 2 - 6 } & MI & 11.8944 & 11.8235 & 11.8971 & 0.0227 \\
\hline \multirow{4}{*}{$\begin{array}{c}\text { Street } \\
\text { Dataset }\end{array}$} & SF & 3.5923 & 2.3690 & 3.5961 & 0.105782 \\
\cline { 2 - 6 } & AG & 30.5306 & 14.5847 & 30.5842 & 0.175562 \\
\cline { 2 - 6 } & ET & 5.9575 & 5.9192 & 5.9575 & 0.191546 \\
\cline { 2 - 6 } & MI & 11.9149 & 11.8383 & 11.9150 & 0.000839 \\
\hline
\end{tabular}

The proposed algorithm accuracy outperforms state-of-theart algorithms in terms of standard evaluation matrices such as Spatial Frequency (SF) and Average Gradients (AG).

\section{CONCLUSION}

A novel approach is suggested in this paper, with a focus to improve the performance of fused image and to highlight the object of image. This algorithm is based on integrating the ADF and DWT. Firstly DWT decomposes the image into four sub bands LL, HL, LH, HH respectively. ADF algorithm is applied on LL sub bands and absolute maximum selection is applied on other three sub bands. Finally perform the inverse DWT of the image is computed to retrieve the fused image. The outcomes attained during the experiment, illustrates that proposed algorithm is better than state-of-the-art algorithm with regards of preserving the edge information of the image. In future, the proposed algorithm can be extended for panchromatic and multispectral image.

\section{ACKNOWLEDGEMENT}

The authors would like to thank CHARUSAT Space Research and Technology Center (CSRTC) for providing platform to conduct the research work.

\section{REFERENCES}

[1] J. Ma, Y. Ma, and C. Li, "Infrared and visible image fusion methods and applications: a survey”, Information Fusion, vol. 45, pp. 153-178, 2019, DOI: https://doi.org/10.1016/j.inffus.2018.02.004.

[2] D. Israni, H. Mewada, "Feature Descriptor Based Identity Retention and Tracking of Players under Intense Occlusion in Soccer Videos", international Journal of Intelligent Engineering and Systems, vol.11, no.4, pp. 31-41, 2018, DOI: 10.22266/ijies2018.0831.04.

[3] D. Israni, H. Mewada, "Identity Retention of Multiple Objects under Extreme Occlusion Scenarios using Feature Descriptors.", vol.14, no.4, pp.290-301, 2018, DOI:https://doi.org/10.24138/jcomss.v14i4.541.

[4] W. da Silva, E.H. Shiguemori, N.L. Vijaykumar, H.F. de Campos Velho, "Estimation of UAV position with use of thermal infrared images.", In IEEE International Conference on Sensing Technology, pp. 828-833, 2015, DOI: 10.1109/ICSensT.2015.7438511.

[5] R. Wu, D. Yu, J. Liu, H. Wu, W. Chen, Q. Gu, "An improved fusion method for infrared and low-light level visible image", In IEEE International Computer Conference on Wavelet Active Media Technology and Information Processing (ICCWAMTIP), pp. 147-151, 2017, DOI: 10.1109/ICCWAMTIP.2017.8301467.

[6] K. Rani, R. Sharma, "Study of different image fusion algorithm", International journal of Emerging Technology and advanced engineering, vol.3, no.5, pp.288-291, 2013.

[7] T.T. Zin, H. Takahashi, T. Toriu, H. Hama, "Fusion of infrared and visible images for robust person detection", Image fusion. IntechOpen, pp. 239-264, 2011

[8] S.S. Bedi, R. Khandelwal, "Comprehensive and comparative study of image fusion techniques", International Journal of Soft Computing and Engineering (IJSCE) ISSN, vol. 3, no. 1, pp.2231-2307, 2013.

[9] Y. Jiang, M. Wang, "Image fusion using multiscale edge-preserving decomposition based on weighted least squares filter", IET image Processing, vol. 8, no. 3, pp. 183-190, 2014, DOI: 10.1049/ietipr.2013.0429.

[10] S. Li, X. Kang, J. Hu, "Image fusion with guided filtering", IEEE Transactions on Image processing, vol. 22, no. 7, pp.2864-2875, 2013, DOI: $10.1109 /$ TIP.2013.2244222.

[11] F. Kahraman, C.D. Mendi, M. Gokmen, "Image frame fusion using 3d anisotropic diffusion", In IEEE International Symposium on Computer and Information Sciences, pp. 1-6, 2008, DOI: 10.1109/ISCIS.2008.4717 968.

[12] B.S Kumar, "Image fusion based on pixel significance using cross bilateral filter", Signal, image and video processing, vol. 9, no. 5, pp.1193-1204, 2015, DOI: https://doi.org/10.1007/s11760-013-05569.

[13] G. Petschnigg, R. Szeliski, M. Agrawala, M. Cohen, H. Hoppe, K. Toyama, "Digital photography with flash and no-flash image pairs", ACM transactions on graphics (TOG), vol. 23, no. 3, pp. 664-672, 2004, doi:10.1145/1015706.1015777.

[14] P. Perona, J. Malik, "Scale-space and edge detection using anisotropic diffusion", IEEE Transactions on pattern analysis and machine intelligence, vol. 12, no. 7, pp. 629-639, 1990, DOI: 10.1109/34.56205.

[15] D.P. Bavirisetti, R. Dhuli, "Fusion of infrared and visible sensor images based on anisotropic diffusion and Karhunen-Loeve transform", IEEE Sensors Journal, vol. 16, no. 1, pp.203-209, 2016, DOI: 10.1109/JSEN.2015.2478655.

[16] L. Deepika, N.M. Mary Sindhuja, "Performance Analysis of Image Fusion Algorithms using HAAR Wavelet", International Journal of Computer Science and Mobile Computing, vol.3, no.1, pp.487-494, 2014.

[17] G Singh, G Singh, GS Aujla, "MHWT-A Modified Haar Wavelet Transformation for Image Fusion", International Journal of Computer Applications, vol.79, no.1, pp.26-31, 2013.

[18] D. Israni, M. Bhatt, "Embedding Color Video Watermark in Image using Orthogonal and Bi-orthogonal Wavelet Transform", Proc. of International Conference on Advances in Computer Science and Application, 2013.

[19] D. Hu, H. Shi, "Fusion of infrared and visible images based on nonsubsampled shearlet transform and block compressive sensing sampling", Ukrainian Journal of Physics, vol.18, no.3, pp.156-167, 2017.

[20] A. Toet, J.K. IJspeert, AM Waxman, M Aguilar, "Fusion of visible and thermal imagery improves situational awareness", Displays, vol.18, no.2, pp.85-95, 1997, DOI: https://doi.org/10.1016/S0141-9382(97)00014-0. 
[21] H. Li, X.J. Wu, J. Kittler, "Infrared and Visible Image Fusion using a Deep Learning Framework", IN International Conference on Pattern Recognition (ICPR), pp. 2705-2710, 2018, DOI: 10.1109/ICPR.2018.85 46006 .

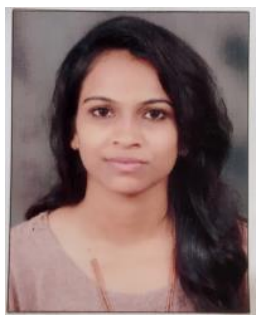

Binal R. Panchotiya is pursuing her Master of Technology degree in U and P U. Patel Department of Computer Engineering, Chandubhai S Patel Institute of Technology, CHARUSAT, Gujarat (INDIA). She has received her Bachelor of Engineering in Computer Engineering from SVBIT, Gandhinagar, Gujarat (India). Her area of interest includes Image Processing, Machine Learning and Internet of Things.

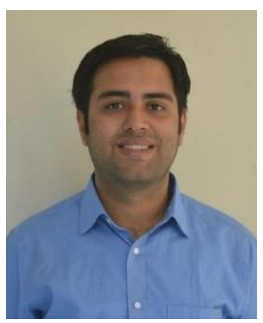

Dippal P. Israni received his M.Tech in Computer Engineering from Dharmsinh Desai University, Gujarat, India. He is currently working as a Lecturer in Information Technology Department, R.C. Technical Institute, Guajrat, India. His major research interests include Computer Vision, Image Processing and Machine Learning. He has 7 years of teaching experience. He has guided $20+$ industry projects at graduate level and 15+ dissertations at Postgraduate Level.

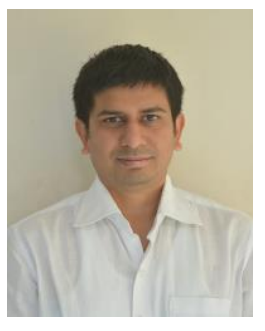

Dr. Ritesh P. Patel has received his Doctorate degree in 2017 from CHARUSAT University. He has received B. Tech. and M. Tech. degrees in 2002 from North Gujarat University and 2004 from DDU respectively. His areas of interest include Cloud Computing, Internet of Things, Communication and Networking, Computer Architecture, Software Engineering and Cluster Computing. He is a member of Professional Societies CSI, IETF. He has 14+ years of teaching experience at UG level and concurrently $07+$ years of teaching and research experience at PG level. He has guided $600+$ students for CCNA. He has guided more than $40+$ industry projects at graduate level and 25+ dissertations at Post Graduate level. 